\title{
Ultrafast structural dynamics of perovskite superlattices
}

\author{
M. Woerner - C. v. Korff Schmising - M. Bargheer • \\ N. Zhavoronkov • I. Vrejoiu • D. Hesse • M. Alexe • \\ T. Elsaesser
}

Received: 26 November 2008 / Accepted: 27 February 2009 / Published online: 10 March 2009

(C) Springer-Verlag 2009

\begin{abstract}
Femtosecond x-ray diffraction provides direct insight into the ultrafast reversible lattice dynamics of materials with a perovskite structure. Superlattice (SL) structures consisting of a sequence of nanometer-thick layer pairs allow for optically inducing a tailored stress profile that drives the lattice motions and for limiting the influence of strain propagation on the observed dynamics. We demonstrate this concept in a series of diffraction experiments with femtosecond time resolution, giving detailed information on the ultrafast lattice dynamics of ferroelectric and ferromagnetic superlattices. Anharmonically coupled lattice motions in a $\mathrm{SrRuO}_{3} / \mathrm{PbZr}_{0.2} \mathrm{Ti}_{0.8} \mathrm{O}_{3}$ (SRO/PZT) SL lead to a switch-off of the electric polarizations on a time scale of the order of 1 ps. Ultrafast magnetostriction of photoexcited SRO layers is demonstrated in a $\mathrm{SRO} / \mathrm{SrTiO}_{3}(\mathrm{STO}) \mathrm{SL}$.
\end{abstract}

PACS 75.80.+q $\cdot$ 61.05.C- $\cdot 78.47 .-\mathrm{p}$

M. Woerner · C. v. Korff Schmising · N. Zhavoronkov ·

T. Elsaesser $(\bowtie)$

Max-Born-Institut für Nichtlineare Optik

und Kurzzeitspektroskopie, Max-Born-Str. 2 A, 12489 Berlin,

Germany

e-mail: elsasser@mbi-berlin.de

M. Bargheer

Institut für Physik und Astronomie, Universität Potsdam, 14476 Potsdam, Germany

I. Vrejoiu · D. Hesse $\cdot$ M. Alexe

Max-Planck-Institut für Mikrostrukturphysik, 06120 Halle, Germany

\section{Introduction}

Polar solids crystallizing in a perovskite structure have received much interest because of their particular structural and electronic properties [1-5]. Electronic and/or spin correlations in such materials give rise to charge ordering, ferroelectricity, superconductivity, ferromagnetism and other phenomena. The macroscopic electric and/or magnetic behavior depends sensitively on the atomic arrangement in the crystal lattice and, thus, can be changed by inducing structural rearrangements, e.g., via built-in or external mechanical stress. This concept has been used to generate materials and/or devices with tailored properties.

So far, perovskite materials have mainly been studied under (quasi)static conditions. Complex phase diagrams and the changes of electronic properties upon changing parameters such as temperature or pressure have been determined by a variety of experimental techniques. Lattice coordinates relevant for ionic ferroelectricity and structure related magnetic properties have been identified for both bulk and nanolayered materials. The latter allow for strain engineering in epitaxial growth and, thus, for tailoring the electronic properties.

Insight into the coupled dynamics of the lattice and the electronic system requires measurements of transient positions of atoms on the intrinsic time scale of their motions in the subpicosecond regime. Here, ultrafast diffraction methods, in particular femtosecond x-ray diffraction, play an important role [6-8]. In ultrafast $\mathrm{x}$-ray diffraction, excitation of the material by a femtosecond optical pulse generates mechanical stress on an ultrashort time scale, in this way driving motions of the lattice. Such motions are probed in real-time by diffracting an ultrashort hard $\mathrm{x}$-ray pulse from the excited sample and measuring the angular position and 
intensity of diffraction peaks as a function of pump-probe delay.

In most cases, the femtosecond excitation pulse interacts with the electronic system of the material and the stress driving the lattice motions is generated more or less impulsively via electron-phonon coupling [9-13]. In bulk materials, the spatial stress profile is mainly determined by the pump beam geometry and the optical penetration depth. The generated lattice elongations cover a broader range of $k$-vectors and the generated strain propagates through the bulk of the excited crystal [14]. In contrast, periodic systems such as superlattices (SLs), i.e., a sequence of nanometerthick layer pairs with layers made from different materials, allow for a spatially distributed and tailored excitation profile by, e.g., exciting only one layer type [15]. This type of excitation is $k$-selective with respect to the so-called superlattice phonon modes, i.e., lattice vibrations affecting the thickness of the SL layers. Moreover, the influence of strain propagation is limited up to picosecond pump-probe delays.

In this report, we discuss ultrafast $\mathrm{x}$-ray diffraction from SLs and demonstrate its potential for elucidating the microscopic lattice dynamics of ferroelectric and ferromagnetic perovskites. The paper is organized as follows. After introducing the basic structural properties of SLs and their consequences for x-ray diffraction (Sect. 2), the experimental techniques applied in our ultrafast diffraction studies are explained in Sect. 3. In Sect. 4, we present results on the lattice dynamics of ferroelectric superlattices, followed by a discussion of ultrafast magnetostriction in Sect. 5. Conclusions and a brief outlook are given in Sect. 6 .

\section{Structural properties of superlattices}

Superlattices consist of a series of nanometer-thick layer pairs which are made from different materials by epitaxial growth or other deposition techniques on a bulk substrate. For layer thicknesses of the order of the electron de Broglie wavelength, the electron wave functions and, thus, the electronic structure of the combined layers are modified compared to their bulk properties $[16,17]$. For instance, minibands for carrier motion along the stacking axis of the layers display a $k$-dispersion different from the bulk materials. Such properties have led to widespread application of semiconductor SL structures with tailored electronic properties, in particular in optoelectronic devices. More recently, SL structures of high structural quality have also been made from perovskite materials applying different methods of synthesis.

From a structural point of view, SLs display periodicities on two different length scales. The periodicity of the crystal lattices of the materials which is characterized by

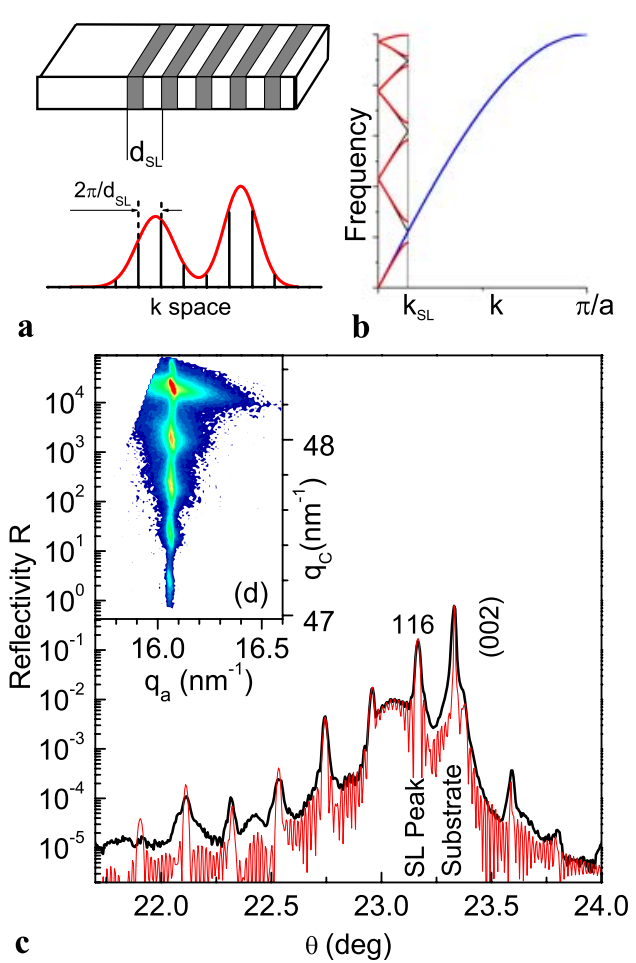

Fig. 1 (a) Upper panel: schematic of a superlattice (SL) geometry consisting of a sequence of layer pairs with a thickness $d_{\mathrm{SL}}$, the SL period. Lower panel: schematic x-ray diffraction pattern from a SL displaying a series of SL Bragg peaks with a constant spacing $g=2 \pi / d_{\mathrm{SL}}$. The envelopes of the Bragg pattern are determined by the structure factors of the two different types of SL layers. (b) Schematic of the SL phonon dispersion. The superlattice period $d_{\mathrm{SL}}$ defines the mini-Brillouin zone with $k_{\mathrm{SL}}=\pi / d_{\mathrm{SL}}$ that is much narrower than the bulk Brillouin zone with $k=\pi / a$ ( $a$ : lattice constant). The acoustic phonon branch of the bulk material (blue line) is folded back into the mini-Brillouin zone, resulting in the dispersion of the SL phonon modes. (c) Static x-ray diffraction pattern of a STO/SRO SL (thick black line) displaying a series of SL peaks and the (002) Bragg peak of the STO substrate. Thin red line: X-ray reflectivity of the sample calculated from dynamical x-ray diffraction theory. (d) Measured reciprocal space map of the SL showing pseudomorphic growth of the sample

the respective sub-nanometer lattice constants, is complemented by the periodicity of the layer sequence with a pe$\operatorname{riod} d_{\mathrm{SL}}$, the nanometer thickness of a layer pair (Fig. 1(a)). The SL periodicity modifies both the spectrum of lattice excitations and the $\mathrm{x}$-ray diffraction patterns. For phonons with $k$-vectors along the SL stack axis, the SL period defines a mini-Brillouin zone of a width $\pm \pi / d_{\mathrm{SL}}$. The corresponding bulk phonon branches are folded back into this minizone as shown schematically for an acoustic phonon branch in Fig. 1(b). The strongly modified dispersion of the backfolded phonons which are connected with elongations of the SL layers along the stack axis, intersects the $k=0$ axis, similar to optical phonons in bulk materials, and develops energy gaps at $k=0$ and $\pm \pi / d_{\mathrm{SL}}[18]$.

In the X-ray Bragg patterns, the SL periodicity leads to the occurrence of equally spaced SL Bragg peaks around the 
bulk Bragg peaks of the layer materials occurring at $2 \pi / a_{A}$ and $2 \pi / a_{B}$ (or multiples) for the materials $\mathrm{A}$ and $\mathrm{B}$ [19]. The spacing of the SL peaks is determined by $g_{\mathrm{SL}}=2 \pi / d_{\mathrm{SL}}$, the intensity envelope of the SL Bragg pattern by the envelopes of the individual SL layers, as shown schematically in Fig. 1(a) (lower panel). In Fig. 1(c), the measured Bragg pattern (thick black line) of a SL sample consisting of 10 pairs of $7.48 \mathrm{~nm}$ thick SRO and $15.24 \mathrm{~nm}$ thick STO layers $\left(d_{\mathrm{SL}}=22.72 \mathrm{~nm}\right)$ displays a series of SL peaks around the (002) bulk peak from the STO substrate. The measured pattern is in very good agreement with calculations based on dynamical diffraction theory (thin red line). The ultrafast response of this sample as monitored via changes of the diffraction pattern will be discussed in Sect. 5 .

\section{Experimental techniques}

The experiments are based on a pump-probe scheme where a sub-100 fs optical pump pulse induces the ultrafast lattice dynamics and a delayed hard $\mathrm{x}$-ray probe pulse is diffracted from the excited sample. Changes of the angular position and intensity of Bragg peaks are measured as a function of pump-probe delay using an x-ray CCD camera for detection.

The experimental setup is based on a Ti:sapphire laser system, providing amplified femtosecond pulses that drive a $\mathrm{Cu} \mathrm{K} \alpha$ plasma source at a $1 \mathrm{kHz}$ repetition rate. Pump pulses in a wavelength range between $800 \mathrm{~nm}$ and $2.2 \mu \mathrm{m}$ were derived from a small part of the output of the Ti:sapphire laser by nonlinear optical frequency conversion. Details of the $\mathrm{x}$ ray source developed at the Max-Born-Institute have been reported elsewhere [20]. Driven by $50 \mathrm{fs}$ pulses of up to $5 \mathrm{~mJ}$ energy, the source provides a total $\mathrm{K}_{\alpha}$ flux (wavelength $0.154 \mathrm{~nm}$ ) of up to $6 \times 10^{10}$ photons/s. The $\mathrm{x}$-ray pulse duration is estimated to be less than $200 \mathrm{fs}$. The pulse-to-pulse intensity fluctuations of the x-ray output are of the order of 10 percent. The $\mathrm{x}$-ray emission originates from a pointlike area of $10 \mu \mathrm{m}$ diameter only, facilitating the imaging of the x-ray output by x-ray optics [21]. In the experiments reported here, the $\mathrm{x}$-ray pulses were focused onto the sample with a multilayer x-ray mirror (Montel optics), resulting in a spot size on the sample of approximately $150 \mu \mathrm{m}$ and an $\mathrm{x}$-ray flux of $10^{6}$ photons/s.

The perovskite SL samples were fabricated by pulsedlaser deposition [22]. The detailed structural parameters are given in Sects. 4 and 5. The samples were placed in a cryostat allowing for a variation of the static sample temperature between 20 and $300 \mathrm{~K}$.

The first ultrafast $\mathrm{x}$-ray diffraction study of a SL was performed with a GaAs/AlGaAs structure consisting of 2000 uncoupled GaAs quantum well layers of $8 \mathrm{~nm}$ thickness separated by $8 \mathrm{~nm}$ thick $\mathrm{Al}_{0.4} \mathrm{Ga}_{0.6}$ As barriers [15]. Femtosecond optical excitation of the GaAs layers launched coherent
SL oscillations periodically modulating the thicknesses of the GaAs and AlGaAs layers at constant $d_{\mathrm{SL}}$. Such oscillations resulted in an intensity modulation of the SL peaks around the (002) Bragg peak of the AlGaAs layer that was measured as a function of pump-probe delay. The modulation of diffracted $\mathrm{x}$-ray intensity originates from a periodic shift of the envelope of the diffraction pattern (cf. Fig. 1(a)) upon periodically changing the AlGaAs lattice constant via the SL oscillations. The phase and amplitude of the modulation demonstrated the displacive character of the mechanism exciting the SL oscillations. Upon electronic excitation, the minimum of the phonon potential energy surface is shifted, resulting in an immediate elongation along the SL oscillation coordinate.

\section{Lattice dynamics of ferroelectric superlattices}

Ferroelectricity in perovskites such as $\mathrm{PbZr}_{x} \mathrm{Ti}_{1-x} \mathrm{O}_{3}$ (PZT) is caused by the spatial displacement of ionic charges in the unit cell (Fig. 2(b)). The lattice parameters and, thus, the ferroelectric properties of thin PZT layers can be changed over a wide range, e.g., by tailoring the strain in the layer [2-4]. The two lattice coordinates essential for the ferroelectric properties are the tetragonality $\eta=c / a$ and the socalled soft mode coordinate $\xi$ (Fig. 2(b)) [23, 24]. The ferroelectric polarization $P$ of the material is proportional to $\xi_{\mathrm{Pb}-\mathrm{Ti}}=\xi_{\mathrm{Pb}-\mathrm{O}}-\xi_{\mathrm{Ti}-\mathrm{O}}$, i.e., $\xi / \xi_{0}=P / P_{0}$ where $\xi_{0}$ and $P_{0}$ are the equilibrium values. The two lattice coordinates are anharmonically coupled through a third-order term in the crystal Hamiltonian proportional to $\eta \xi_{\mathrm{Pb}-\mathrm{Ti}}^{2}$. As a result, changes of the tetragonality $\eta$ affect the ferroelectric polarization directly, a mechanism that allows for switching the ferroelectric properties by transiently manipulating the lattice along $\eta$.

In this section, we present recent results on the ultrafast lattice dynamics of a PZT/SRO SL (Fig. 2(a)) [25]. The SL was grown on a STO substrate and consists of a $60 \mathrm{~nm}$ thick SRO bottom layer followed by 15 pairs of PZT
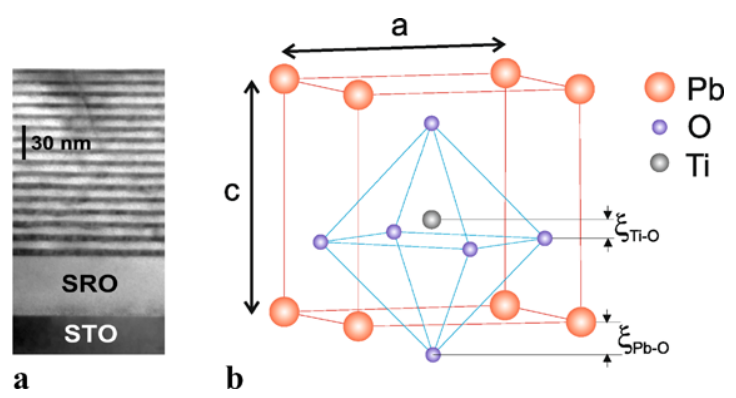

Fig. 2 (a) Transmission electron micrograph of a thinned PZT/SRO superlattice. (b) Unit cell of PZT with the relevant coordinates, the tetragonal distortion $\eta=c / a$ and the ferroelectric ion displacements $\xi_{\mathrm{Pb}-\mathrm{O}}$ and $\xi_{\mathrm{Ti}-\mathrm{O}}$ 

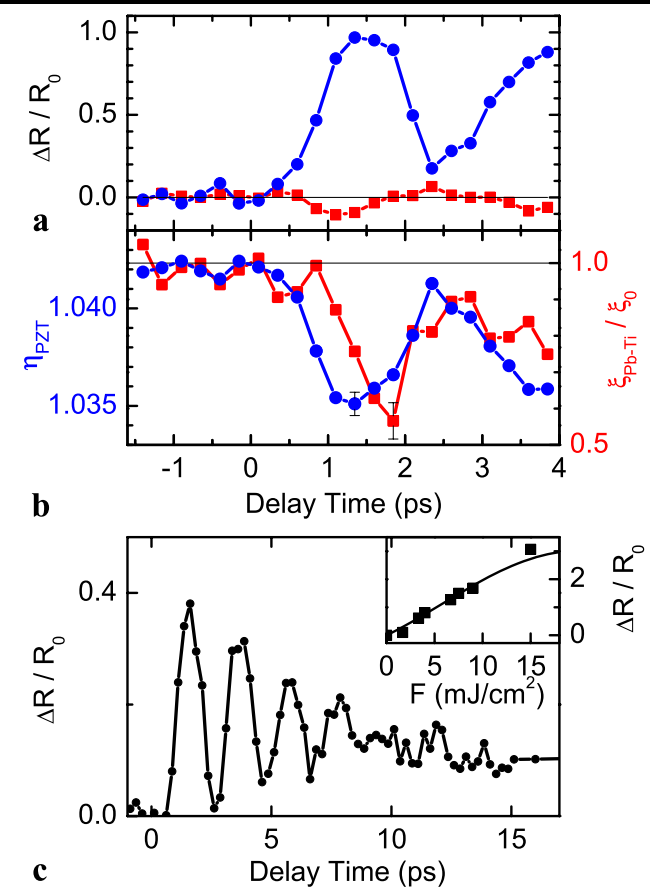

Fig. 3 (a) Transient intensity changes of the (0 0 56) (blue circles) and (0 0 55) (red squares) SL Bragg peaks. The normalized change of $\mathrm{x}$-ray reflectivity $\Delta R / R_{0}=\left(R-R_{0}\right) / R_{0}$ is plotted versus the pump-probe delay for a pump fluence of $5 \mathrm{~mJ} / \mathrm{cm}^{2}$. (b) Transient change of the tetragonality $\eta_{\mathrm{PZT}}$ (circles) and normalized soft mode elongation $\xi / \xi_{0}$ (squares) as derived from the data in (a). (c) Oscillations of the $\left(\begin{array}{ll}0 & 0\end{array} 56\right)$ $\mathrm{x}$-ray reflectivity on an extended time scale. Inset: maximum amplitude of the reflectivity change as a function of pump fluence

$\left(d_{\mathrm{PZT}}=4.92 \mathrm{~nm}\right)$ and SRO $\left(d_{\mathrm{SRO}}=6.29 \mathrm{~nm}\right)$ layers. Such layers were grown with their c axis (Fig. 2(b)) parallel to the stack axis. In the femtosecond experiments, a 50 fs pump pulse at $800 \mathrm{~nm}$ excites the metallic SRO layers, in this way generating an electronic excitation with a spatial period of $d_{\mathrm{SL}}=d_{\mathrm{PZT}}+d_{\mathrm{SRO}}$. The coupling of the photoexcited carriers to the lattice via electron-phonon interaction generates transient stress along the $\mathrm{c}$ axis of the PZT and SRO layers and the resulting SL dynamics are monitored by diffracting hard $\mathrm{x}$-ray pulses from the excited sample. To get insight into lattice motions along both relevant coordinates $\eta$ and $\xi$ (cf. Fig. 2(b)), intensity changes of the (0 0056$)$ and $\left(\begin{array}{lll}0 & 0 & 55\end{array}\right)$ SL Bragg peaks were measured. As discussed in Ref. [25], the $(0056)$ peak is located at a Bragg angle where the slope of the PZT and SRO envelope functions is substantial, making the intensity of this peak most sensitive to angular shifts of the envelopes by transient changes of the individual layer thicknesses. In this way, motions along the coordinate $\eta$ become visible directly. In contrast, the $\left(\begin{array}{lll}0 & 0 & 55\end{array}\right)$ reflection is located at the maximum of the PZT envelope function and its intensity is particularly sensitive to the ionic displacement $\xi_{\mathrm{Pb}-\mathrm{Ti}}$ within the PZT unit cell.

In Fig. 3(a), the change of the $\mathrm{x}$-ray reflectivity $\Delta R / R_{0}$ of the (0 0 56) (circles) and (0 0 55) (squares) Bragg peaks
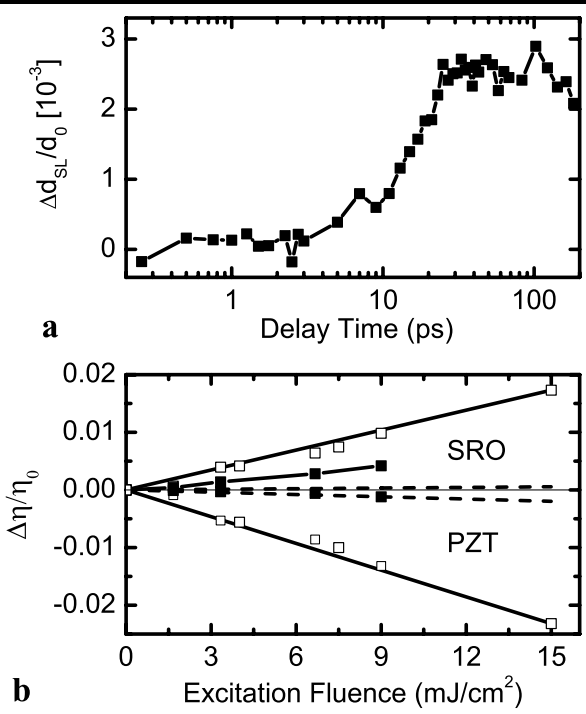

Fig. 4 (a) Change of the superlattice period $\Delta d_{\mathrm{SL}} / d_{0}$ as a function of delay time as derived from the angular positions of the Bragg peaks ( $d_{0}$ : period of the unexcited SL). (b) Change of tetragonality $\Delta \eta / \eta_{0}$ of the SRO and PZT layers after 1.5 ps (open symbols) and 200 ps (solid symbols). The solid lines are guides to the eye. Dashed lines: calculated changes of the SRO and PZT tetragonalities due to thermal expansion and compression

are plotted as a function of pump-probe delay (pump fluence $5 \mathrm{~mJ} / \mathrm{cm}^{2}$ ). The reflectivity changes display a delayed rise, followed by oscillations. In Fig. 3(c), such oscillations are shown on an extended time scale (pump fluence $2 \mathrm{~mJ} / \mathrm{cm}^{2}$ ). In addition to changes of the diffracted intensity, the timedependent angular positions of the SL Bragg peaks were studied. In Fig. 4(a), the change of the SL period derived from the position of the ( 0056$)$ peak is plotted up to pumpprobe delays of $200 \mathrm{ps}$ (logarithmic time scale). During the first 4 ps, the SL period is constant, followed by a pronounced increase between 10 and $30 \mathrm{ps,} \mathrm{and} \mathrm{a} \mathrm{(larger)} \mathrm{con-}$ stant value at longer time delays.

The reflectivity changes on the ( $\left.\begin{array}{lll}0 & 56\end{array}\right)$ and $\left(\begin{array}{lll}0 & 0 & 55\end{array}\right) \mathrm{SL}$ Bragg peaks are driven by the photogenerated stress along the $\mathrm{c}$ axis of the PZT and SRO layers. This stress rises with a time constant of approximately $500 \mathrm{fs}$, reflecting the transformation of the initial electronic into a lattice excitation as be will discussed in more detail in Sect. 5. In this process, a small fraction of the excitation energy is converted into a coherent SL excitation along the lattice coordinate $\eta$. The stress generates an oscillatory motion of the SL as is evident from the reflectivity oscillations of Figs. 3(a, c). The oscillations are due to the coherent superposition of SL phonon states (cf. Fig. 1(b)) in a wave packet periodically modulating the tetragonality $\eta$ of the PZT and SRO layers. The 2.2 ps period of the oscillations is determined by $d_{\mathrm{SL}}$ and the velocity of sound in the material.

The anharmonic coupling of the directly driven tetragonal distortion $\eta$ and the soft mode coordinate $\xi$ results in 
an elongation along $\xi$ and a corresponding change of the ferroelectric polarization $P$. The intensities of the two SL Bragg peaks studied in our measurements behave differently as a function of the two coordinates. As a result, the timedependent elongations along the two coordinates $\xi$ and $\eta$ can be derived in a quantitative way from the data recorded during the first $4 \mathrm{ps}$ when the SL period is constant. The time evolutions of $\eta_{\mathrm{PZT}}$ and $\xi / \xi_{0}=P / P_{0}$ are shown in Fig. 3(b). The tetragonal distortion of PZT reaches a maximum after 1.3 ps. The soft mode coordinate $\xi / \xi_{0}$ shows an ultrafast and slightly delayed reduction, corresponding to a decrease of the polarization $P / P_{0}$ of approximately $50 \%$. With the highest excitation fluence in our experiments of $15 \mathrm{~mJ} / \mathrm{cm}^{2}$ (inset of Fig. 3(c)), we generated a peak strain on the PZT layers of $\Delta \eta / \eta_{0}=2.3 \%$. This strain leads to a complete switch-off of the polarization $P\left[\xi / \xi_{0}=0\right]$ on a time scale of a few picoseconds. Such ultrafast switching may find application in future ferroelectric device structures.

At pump-probe delays longer than 4 ps, the SL period increases (Fig. 4(a)). This expansion originates from the unbalanced stress at the interfaces of the SL with the substrate and with air [14] and the resulting strain propagates through the entire SL structure. One expects the SL Bragg peaks to shift on a time scale $T=N \cdot d_{\mathrm{SL}} / v_{\mathrm{ph}} \approx 30 \mathrm{ps}$, where $N=15$ is the number of SL layer pairs and $v_{\mathrm{ph}} \approx 5 \mathrm{~nm} / \mathrm{ps}$ the velocity of sound, a behavior borne out by the data of Fig. 4(a). A simultaneous analysis of the reflectivity change and the change of the SL period $\Delta d_{\mathrm{SL}} / d_{0}$ of the $\left(\begin{array}{lll}0 & 0 & 56\end{array}\right)$ Bragg peak allows one to determine the tetragonal distortion $\eta$ of the PZT and SRO layers separately. In Fig. 4(b), the change $\Delta \eta / \eta_{0}$ after $1.5 \mathrm{ps}$ and $200 \mathrm{ps}$ is plotted as a function of excitation fluence, displaying a linear dependence. After 200 ps, the metallic SRO layers are still expanded by approximately $50 \%$ of the peak value, which is an order of magnitude larger than the estimated thermal expansion. The calculated change of $\eta$ due to an estimated temperature rise of $30 \mathrm{~K}$ is plotted in Fig. 4(b) as a dashed line. In contrast, the PZT layers show a much smaller compression after 200 ps which can be explained by the induced temperature rise and the negative thermal expansion coefficient of PZT (dashed line in Fig. 4(b)). This implies that the tetragonal distortion $\eta_{0}$ is restored after $30 \mathrm{ps}$ and the recovery of the PZT polarization $\left(\xi_{\mathrm{Pb}-\mathrm{Ti}}(30 \mathrm{ps}) \approx \xi_{0}\right)$ is directly connected to the expansion of the entire SL.

\section{Ultrafast magnetostriction in SRO}

So far, we have discussed the correlation of ionic charges in a crystal lattice leading to ferroelectricity. Magnetism is determined by correlation of electron spins via the exchange interaction, another basic type of charge correlation in solids. The Stoner model [26] represents an elementary a

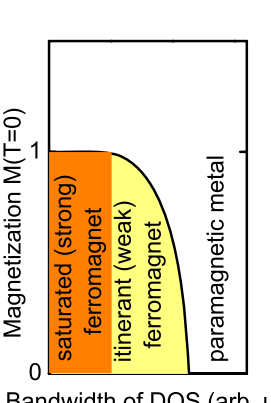

b Ferromagnetic

Paramagnetic

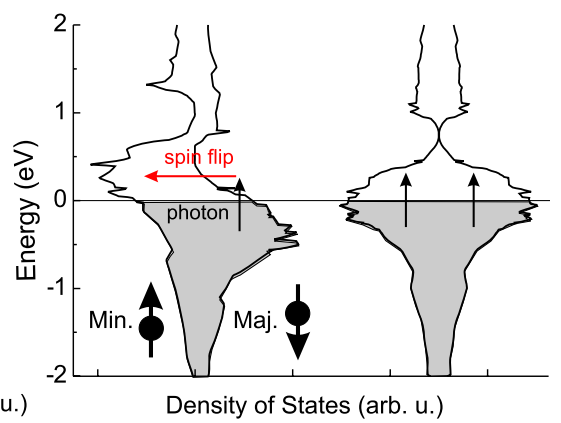

Fig. 5 (a) Schematic of the macroscopic magnetization as a function of the energy width of the density of states (DOS) according to the Stoner model. (b) Schematic of the density of states of minority and majority spins in SRO for the ferro- and the paramagnetic phase

approach which describes the magnetic properties via the interplay of exchange and kinetic energy of electrons in a partially filled conduction band. In a ferromagnet, the net excess of electrons with spins pointing into one direction gives rise to the macroscopic magnetization. Compared to a Fermi distribution with equal populations in both spin directions, there is a net reduction of the total electron energy determined by the decrease of the total exchange energy minus the increase in the total kinetic energy. This behavior is particularly pronounced for an exchange energy large compared to the energetic width of the conduction band, the latter representing the upper limit for the kinetic energy of the carriers (Fig. 5(a)). With increasing kinetic energy of the electron gas, i.e., increasing width of the conduction band, the net energy gain for parallel spins and, thus, the ferromagnetic magnetization become smaller. In this range, the weak socalled itinerant ferromagnetism depends sensitively on the details of the band structure and the density of conduction band states which in turn are determined by the lattice geometry, e.g., via the overlap of atomic orbitals. For large kinetic energies, both spin directions show similar populations and the system becomes paramagnetic.

SRO is a prototype material displaying itinerant ferromagnetism below a Curie temperature $T_{C}=160 \mathrm{~K}$ [27-30]. The calculated density of states and a schematic of the electron distribution in the ferro- and paramagnetic phase are shown in Fig. 5(b). The hybridization of the 4 d orbitals of the $\mathrm{Ru}$ ions with the $2 \mathrm{p}$ orbitals of the oxygen atoms that are arranged on octaeders around the Ru sites, plays a key role for the electronic properties of SRO. The interplay between ferromagnetism and the lattice geometry is also manifested in the invar effect of SRO, i.e., the temperature-independent volume of the crystal below $T_{C}[31,32]$. In this regime, the positive thermal expansion due to anharmonic phononphonon interactions is compensated by a negative magnetostrictive contribution $[33,34]$.

In the following, we present the first study addressing the ultrafast lattice response in the ferro- and paramag- 
netic phase of SRO after excitation of the electronic system by a sub-100 fs pulse at different spectral positions (cf. Fig. 7(b)) [35]. Such excitation generates a uniaxial stress inducing lattice motions of the SRO layers. The analysis of such motions provides insight into the dynamics and the mechanisms of stress generation.

The SL consists of 10 double layers of STO/SRO on a $50 \mathrm{~nm}$ thick SRO buffer layer grown on a STO substrate. The equilibrium structure of the sample was derived from the static x-ray characterization summarized in Fig. 1(c, d). Figure 1(d) shows a high resolution reciprocal space map around the asymmetric Bragg reflection (1 03 ) of the STO substrate. The intensity of the STO substrate and SL Bragg peaks are plotted on a logarithmic scale as a function of the in- and out-of-plane reciprocal lattice vectors $q_{a}$ and $q_{c}$. The map demonstrates pseudomorphic growth with identical in-plane lattice constants $a$ of the SL structure and the STO substrate. From the Bragg pattern in Fig. 1(c) which was analyzed by dynamic diffraction theory, one derives the following sample parameters: $c_{\mathrm{STO}}=0.3907 \mathrm{~nm}$, $c_{\mathrm{SRO}}=0.3938 \mathrm{~nm}, d_{\mathrm{STO}}=15.24 \mathrm{~nm}$ and $d_{\mathrm{SRO}}=7.48 \mathrm{~nm}$ with $d_{\mathrm{SL}}=d_{\mathrm{STO}}+d_{\mathrm{SRO}}$.

The fully reversible transient change of x-ray intensity $\Delta R / R_{0}$ reflected from the sample at room temperature after excitation of the SRO layers at different spectral positions is plotted in Figs. 6(a, b) (pump fluence $6.5 \mathrm{~mJ} / \mathrm{cm}^{2}$ ). The transients show a delayed rise and oscillations with a period of 3 ps. Zero time delay is measured with an accuracy of $\pm 100 \mathrm{~s}$ using an all-optical reflectivity measurement in the same setup (solid line in Fig. 6(b)). We observe the same oscillation amplitude and time evolution of the $\mathrm{x}$-ray reflectivity change for identical pump fluences absorbed at the different excitation wavelengths.

$\mathrm{X}$-ray reflectivity data for a wide range of sample temperatures and a pump wavelength of $2.2 \mu \mathrm{m}$ are presented in Fig. 7. The transients in Fig. 7(c) show a temperatureindependent time evolution, i.e., identical initial delay, oscillation period and phase. The low-temperature amplitude is, however, less than 50 percent of that at room temperature. Measurements with sample temperatures between 20 and $300 \mathrm{~K}$ and identical pump fluences reveal a continuous increase of oscillation amplitude up to $T_{C}=160 \mathrm{~K}$ (Fig. 7(a), diamonds). A similar though weaker variation of the signal amplitudes is found after excitation at $800 \mathrm{~nm}$ (circles).

In our experiments, the femtosecond pump pulse induces an electronic excitation of the SRO layers which generates a mechanical stress of the lattice via the coupling of the electronic system to lattice degrees of freedom. In our excitation scheme, this uniaxial stress is spatially periodic and drives a spatially periodic strain wave, changing the thicknesses of the SRO/STO layers and the intensity of the (lo 0 116) SL Bragg peak periodically. The unchanged angular position of the Bragg peak shows that this oscillation along the c-axes of

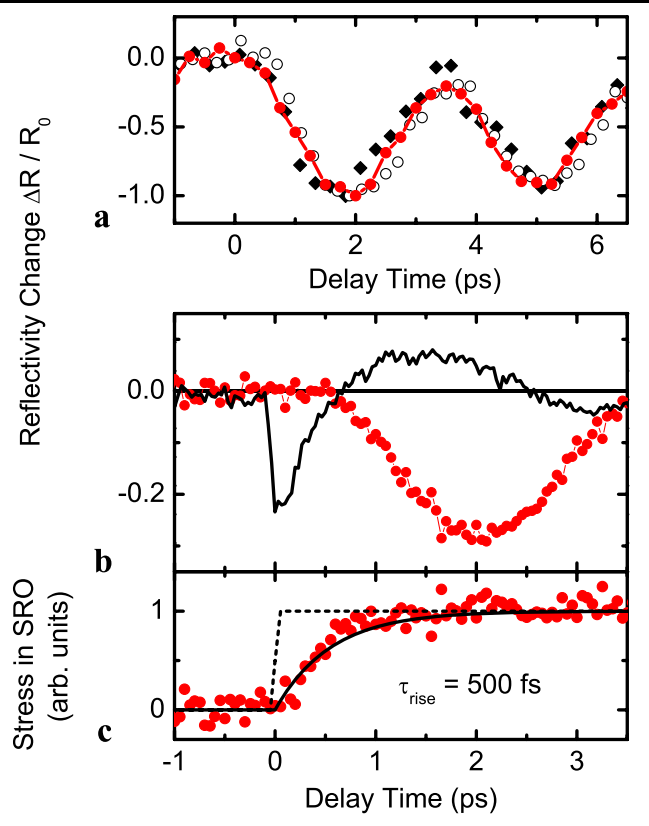

Fig. 6 (a) Normalized change of x-ray reflectivity of the SRO/STO SL as a function of pump-probe delay for excitation at $0.8 \mu \mathrm{m}$ (solid circles), $1.28 \mu \mathrm{m}$ (diamonds), and $2.2 \mu \mathrm{m}$ (open circles). Amplitudes and time evolution are identical for the same absorbed pump fluence of $6.5 \mathrm{~mJ} / \mathrm{cm}^{2}$. (b) Time resolved change of $\mathrm{x}$-ray reflectivity after excitation at $0.8 \mu \mathrm{m}$ (symbols). Solid line: optical reflectivity from a $0.8 \mu \mathrm{m}$ pump/0.8 $\mu \mathrm{m}$ probe study defining delay zero. (c) Time evolution of the uniaxial stress in the SRO layers derived from the data in (b) (symbols). Solid line: fit with a $500 \mathrm{fs}$ rise time. Dashed line: instantaneous response

the SRO and STO lattice occurs at a constant SL period $d_{\mathrm{SL}}$, similar to the behavior discussed in Sect. 4 .

The delayed onset of the oscillations originates from the finite build-up time of the uniaxial stress after electronic excitation. For a quantitative analysis, the driving force $F(t)$ was derived from a fit to the data based on the differential equation $d^{2} \Delta d_{\mathrm{SRO}} / d t^{2}-\omega_{0}^{2} \Delta d_{\mathrm{SRO}}=F(t)$ with the SL oscillation frequency $\omega_{0}$. The force $F(t)$ (Fig. 6(c)) shows a noninstantaneous rise with a time constant of $\tau_{\text {rise }}=500 \mathrm{fs}$, independent of excitation wavelength and sample temperature. The subpicosecond rise time rules out a direct, e.g., displacive stress generation via electronic excitation. The uniaxial stress is due to a nonequilibrium phonon population generated by electron cooling and, in the ferromagnetic phase, to magnetostriction. Carrier cooling in SRO as in other metals, occurs on a subpicosecond time scale which has been studied with our sample in independent all-optical experiments [35].

In the paramagnetic phase $\left(T>T_{C}\right)$, the nonequilibrium phonon population generates strain with an amplitude determined by the amount of pump energy deposited and a temperature-independent amplitude (Fig. 7(a)). In the ferromagnetic phase $\left(T<T_{C}\right)$, the optical excitation induces spin-flip processes (Fig. 7(b)) and, thus, both phonon generated and magnetostrictive stress components of opposite 


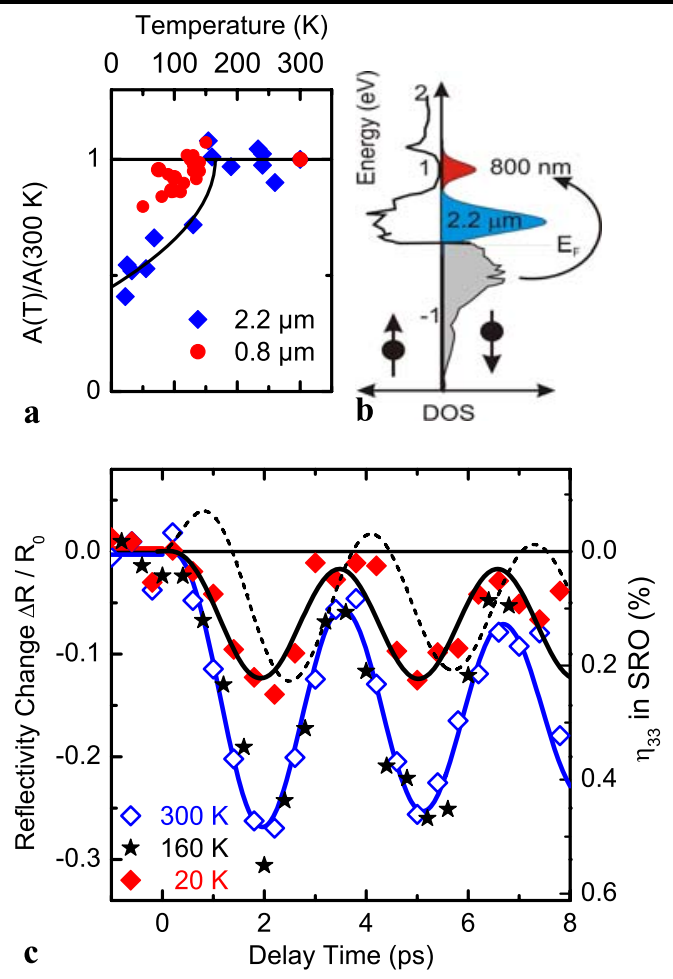

Fig. 7 (a) Maximum amplitude of the reflectivity change $A(T)$ at a sample temperature $T$ normalized to $A(300 \mathrm{~K})$ as a function of temperature. Solid line: temperature-dependent magnetization squared. (b) Schematic of the femtosecond optical excitation promoting carriers into unoccupied states from where spin flips occur. (c) Transient $\mathrm{x}$-ray reflectivity for different initial sample temperatures (symbols, excitation wavelength $2.2 \mu \mathrm{m}$ ). Dashed line: response calculated with an instantaneous rise of magnetostrictive and a delayed rise of phonon induced stress

sign occur. Their superposition leads to the pronounced change of the strain amplitude with temperature and excitation wavelength (Fig. 7(a)). The amplitude change with temperature mimics the temperature-dependent magnetization $M(T)^{2}$ (solid line in Fig. 7(a)). The amplitude reduction is caused by the contractive magnetic contribution to the stress driving the SL oscillations. Such a contribution originates from the strong magneto-volume effect present in bulk [36] and nanolayered SRO [37]. At $T=20 \mathrm{~K}$, the magnetostriction reaches a very high value of approximately $\eta_{\text {mag }}=2.5 \times 10^{-3}$, in quantitative agreement with the expected magnitude for SRO nanolayers grown on STO substrate [37].

The buildup kinetics of stress is identical in the paramagnetic and ferromagnetic phase as is the phase of the SL oscillations [35]. Thus, the rise time $\tau_{\mathrm{mag}}$ of the magnetostrictive stress is very similar to that of the nonequilibrium phonon contribution, i.e., $200 \mathrm{fs}<\tau_{\mathrm{mag}}<700 \mathrm{fs}$. A rise of magnetostriction on a sub-100 fs time scale can be ruled out as is evident from a simulation of SL oscillations driven by an instantaneous magnetostrictive and a delayed phonon mediated stress (dashed line in Fig. 7(c)).

\section{Conclusions and outlook}

The results presented here demonstrate the potential of ultrafast $\mathrm{x}$-ray diffraction to unravel reversible lattice motions in real-time and to provide detailed information on the underlying anharmonic couplings within the lattice and the coupling of the lattice motions to elementary excitations of the electronic system. The application of superlattice structures for such studies allows for a spatially distributed tailored excitation and generation of mechanical stress, resulting in lattice motions with particular $k$-vectors. Beyond the perovskite superlattices studied here, we envisage the extension of this concept to a much wider class of materials, in particular to charge and spin correlated systems relevant for applications in (opto)electronics and magnetism.

Acknowledgements We would like to acknowledge the important contributions of our present and former coworkers Y. Gritsai, M. Kiel, A. Harpoeth, and Z. Ansari to the results reported here. We thank M. Zamponi, I. Uschmann, and E. Förster, Universität Jena, for joint work on the X-ray source, and M. Schmidbauer, Leibniz Institute for Crystal Growth, Berlin, for his help in sample characterization. This work has been supported by the Deutsche Forschungsgemeinschaft through the Schwerpunktprogramm 1134.

\section{References}

1. T. Wolfram, S. Ellialtioglu, Electronic and Optical Properties of d-Band Perovskites (Cambridge, 2006)

2. C.H. Ahn, K.M. Rabe, J.M. Triscone, Science 303, 488 (2004)

3. R.A. McKee, F.J. Walker, M.F. Chisholm, Science 293, 468 (2001)

4. J.F. Scott, C.A. Paz de Araujo, Science 246, 1400 (1989)

5. M. Dawber, K.M. Rabe, J.F. Scott, Rev. Mod. Phys. 77, 1083 (2005)

6. A. Rousse, C. Rischel, J.C. Gauthier, Rev. Mod. Phys. 73, 17 (2001)

7. M. Bargheer, N. Zhavoronkov, M. Woerner, T. Elsaesser, Chem. Phys. Chem. 7, 783 (2006)

8. C. v. Korff Schmising, M. Bargheer, M. Woerner, T. Elsaesser, Z. Kristallogr. 223, 283 (2008)

9. K. Sokolowski-Tinten, C. Blome, C. Dietrich, A. Tarasevitch, M. Horn von Hoegen, D. von der Linde, A. Cavalleri, J. Squier, M. Kammler, Phys. Rev. Lett. 87, 225701 (2001)

10. K. Sokolowski-Tinten, C. Blome, J. Blums, A. Cavalleri, C. Dietrich, A. Tarasevitch, I. Uschmann, E. Förster, M. Kammler, M. Horn von Hoegen, D. von der Linde, Nature 422, 287 (2003)

11. J.L.A.M. Lindenberg et al., Science 308, 392 (2005)

12. C. Rose-Petruck, R. Jiminez, T. Guo, A. Cavalleri, C. Siders, F. Raksi, J.A. Squier, B.C. Walker, K.R. Wilson, C.P.J. Barty, Nature 398, 310 (1999)

13. A.M. Lindenberg, I. Kang, S.L. Johnson, T. Misalla, P.A. Heimann, Z. Chang, J. Larsson, P.H. Bucksbaum, H.C. Kapteyn, H.A. Padmore, R.W. Lee, J.S. Wark, R.W. Falcone, Phys. Rev. Lett. 84, 111 (2000)

14. C. v. Korff Schmising, M. Bargheer, M. Kiel, N. Zhavoronkov, M. Woerner, T. Elsaesser, Phys. Rev. B 73, 212202 (2006)

15. M. Bargheer, N. Zhavoronkov, Y. Gritsai, J.C. Woo, D.S. Kim, M. Woerner, T. Elsaesser, Science 306, 1771 (2004)

16. M. Helm, Semicond. Sci. Technol. 10, 557 (1995), and references therein 
17. J. Shah, Ultrafast Spectroscopy of Semiconductors and Semiconductor Nanostructures, 2nd edn. (Springer, Berlin, 1999)

18. C. Colvard, T.A. Gant, M.V. Klein, R. Merlin, R. Fischer, H. Morkoc, A.C. Gossard, Phys. Rev. B 31, 2080 (1985)

19. A. Krost, G. Bauer, J. Woitok, in Optical Characterization of Epitaxial Semiconductor Layers, ed. by G. Bauer, W. Richter (Springer, Berlin, 1996), pp. 287-391

20. N. Zhavoronkov, Y. Gritsai, M. Bargheer, M. Woerner, T. Elsaesser, F. Zamponi, I. Uschmann, E. Förster, Opt. Lett. 30, 1737 (2005)

21. M. Bargheer, N. Zhavoronkov, N. Bruch, H. Legall, H. Stiel, M. Woerner, T. Elsaesser, Appl. Phys. B 80, 715 (2005)

22. I. Vrejoiu, G. Le Rhun, L. Pintilie, D. Hesse, M. Alexe, U. Gösele, Adv. Mater. 18, 1657 (2006)

23. C. Lichtensteiger, J.M. Triscone, J. Junquera, P. Ghosez, Phys. Rev. Lett. 94, 047603 (2005)

24. J.A. Sanjurjo, E. Lopez-Cruz, G. Burns, Solid State Commun. 48, $221(1983)$

25. C. v. Korff Schmising, M. Bargheer, M. Kiel, N. Zhavoronkov, M. Woerner, T. Elsaesser, I. Vrejoiu, D. Hesse, M. Alexe, Phys. Rev. Lett. 98, 257601 (2007)

26. E.C. Stoner, Proc. R. Soc. Lond. A 165, 372 (1938)

27. I.I. Mazin, D.J. Singh, Phys. Rev. B 56, 2556 (1997)
28. J.S. Lee, Y.S. Lee, T.W. Noh, S. Nakatsuji, H. Fukuzawa, R.S. Perry, Y. Maeno, Y. Yoshida, S.I. Ikeda, J. Yu, C.B. Eom, Phys. Rev. B 70, 085103 (2004)

29. K. Fujioka, J. Okamoto, T. Mizokawa, A. Fujimori, I. Hase, M. Abbate, H.J. Lin, C.T. Chen, Y. Takeda, M. Takano, Phys. Rev. B 56, 6380 (1997)

30. P.B. Allen, H. Berger, O. Chauvet, L. Forro, T. Jarlborg, A. Junod, B. Revaz, G. Santi, Phys. Rev. B 53, 4393 (1996)

31. R.J. Weiss, Proc. Phys. Soc. Lond. 82, 281 (1963)

32. J.L. Robertson, G.E. Ice, C.J. Sparks, X. Jiang, P. Zschack, F. Bley, S. Lefebvre, M. Bessiere, Phys. Rev. Lett. 82, 2911 (1999)

33. L.W. McKeehan, P.P. Cioffi, Phys. Rev. 28, 146 (1926)

34. S.C. Masmanidis, H.X. Tang, E.B. Myers, M. Li, K. De Greve, G. Vermeulen, W. Van Roy, M.L. Roukes, Phys. Rev. Lett. 95, $187206(2005)$

35. C. v. Korff Schmising, A. Harpoeth, N. Zhavoronkov, Z. Ansari, C. Aku-Leh, M. Woerner, T. Elsaesser, M. Bargheer, M. Schmidbauer, I. Vrejoiu, D. Hesse, M. Alexe, Phys. Rev. B 78, 060404(R) (2008)

36. T. Kiyama, K. Yoshimura, K. Kosuge, Y. Ikeda, Y. Bando, Phys. Rev. B 54, R756 (1996)

37. O. Gan, R.A. Rao, C.B. Eom, J.L. Garrett, M. Lee, Appl. Phys. Lett. 72, 978 (1998) 